Chirurg 2021 · 92:274-280 https://doi.org/10.1007/s00104-020-01276-7 Online publiziert: 21 . September 2020 (c) Der/die Autor(en) 2020
Nils Mühlenfeld · Cora R. Schindler · Jasmina Sterz • Philipp Thönissen · Philipp Störmann · Ingo Marzi · René D. Verboket

Goethe Universität Frankfurt, Frankfurt am Main, Deutschland

\title{
Kosten-Erlös-Defizit der ambulanten Versorgung von Kopfplatzwunden in der Notaufnahme
}

heutzutage zunehmend überfüllten Notaufnahme eine Ressourcenbindung über einen längeren Zeitraum, der nicht selten in deutlich verlängerten Wartezeiten für Folgepatienten resultieren kann. Dies gilt insbesondere bei der Versorgung intoxikierter und/oder dementer Patienten. Auch die regelmäßige Nachsorge zur Prävention einer Infektion oder Nahtdehiszenz ist ein nicht $\mathrm{zu}$ vernachlässigender Zeitaufwand für die Krankenhaussprechstunde oder den niedergelassenen Kollegen. Die adäquate Versorgung ist jedoch für das Wohlergehen der Patienten von großer Bedeutung. Leider führt die Vergütung nach einheitlichem Bewertungsmaßstab (EBM) oft zu einer unzureichenden Vergütung der Notfallleistungen und $\mathrm{zu}$ einer regelhaften Kostenunterdeckung in den Notaufnahmen [7, 17].

Ziel dieser Arbeit ist es, die Ätiologie von Kopfplatzwunden in einer großen Notaufnahme zu beschreiben und das Kosten-Erlös-Verhältnis der ambulanten Wundversorgung von Kopfverletzungen anhand der Vergütung von Notfallleistungen auf der Kalkulationsgrundlage des EBM zu untersuchen und $\mathrm{zu}$ evaluieren, ob die im EBM gegebene $\mathrm{Ab}$ rechnungsgrundlage mit den auflaufenden Kosten im Einklang steht.

\section{Studiendesign und Methoden}

\section{Studiendesign und Datenakquise}

Die Patientenzahl enthält ausschließlich gesetzlich Versicherte. Selbstzahler, Pri- vatpatienten und berufsgenossenschaftliche Fälle wurden aufgrund anderer Abrechnungsmodalitäten und sehr geringer Fallzahlen ausgeschlossen. Die Daten der insgesamt 724 Fälle wurden in der Zentralen Notaufnahme (ZNA) am Universitätsklinikum Frankfurt am Main im Jahr 2018 erhoben. Mittels einer systematischen Abfrage im Krankenhausinformationssystem (Hospital Information System [HIS]) wurden alle Patienten identifiziert, die in diesem Zeitraum eine Wundversorgung einer Kopfplatzwunde mittels Naht (EBM 02301) erhielten. Fälle mit unklarer Art der Wundversorgung oder unzureichender Dokumentation wurden ausgeschlossen, auch Kinder, bei denen Kopfwunden häufig geklebt werden können, wurden ausgeschlossen. Die Genehmigung der Studie erfolgte unter dem Ethikvotum Nummer 19-491 der Universitätsklinik Frankfurt, die vorliegende Studie folgt den STROBE(Strengthening The Reporting of Observational Studies in Epidemiology)-Richtlinien für Beobachtungsstudien sowie den RECORD(Reporting of studies Conducted using Observational Routinely-collected Data)-Richtlinien für Observationsstudien $[4,6]$.

\section{Datenerhebung und statistische Analyse}

Die Berechnung der Erlöse erfolgte anhand der einheitlich abgerechneten Notfallpauschale EBM 01210 bei Inanspruchnahme von Montag bis Freitag zwischen 07:00 und 19:00 Uhr und der 
Tab. 1 Kosten für die Wundversorgung einer Kopfplatzwunde mittels Naht

\begin{tabular}{|c|c|c|}
\hline Verbrauchsmaterial & Anzahl & Kosten \\
\hline Wundversorgungsset, steril (Einmalinstrumente) & 1 & $9,30 €$ \\
\hline Tupfer, steril (3er Pack) & 1 & $0,21 €$ \\
\hline Abdecktuch, steril & 1 & $0,46 €$ \\
\hline Lochtuch, steril & 1 & $0,57 €$ \\
\hline Haube & 1 & $0,05 €$ \\
\hline Mundschutz & 2 & $0,07 €$ \\
\hline Handschuhe, steril & 1 & $0,31 €$ \\
\hline Nahtmaterial & 1 & $3,91 €$ \\
\hline Einmalskalpell, steril & 1 & $3,50 €$ \\
\hline Spritze $10 \mathrm{ml}$, steril & 2 & $0,08 €$ \\
\hline Kanüle, steril & 2 & $0,02 €$ \\
\hline Kompressen, steril & 2 & $0,06 €$ \\
\hline Mullbinde & 1 & $0,07 €$ \\
\hline $\mathrm{NaCl} 0,9 \% 10 \mathrm{ml}$ & 2 & $0,06 €$ \\
\hline $\begin{array}{l}\text { Desinfektion (z. B. Octenisept }{ }^{\circ} \text { (Octenidindihydrochlorid), Braunol }{ }^{\circ} \\
\text { (PVP-lod)) }\end{array}$ & $300 \mathrm{ml}$ & $1,78 €$ \\
\hline Lokalanästhetikum (z. B. Mecain ${ }^{\circledR}$ (Mepivacain)) & $5 \mathrm{ml}$ & $0,29 €$ \\
\hline \multicolumn{3}{|l|}{ Summe Material: $20,74 €$} \\
\hline \multicolumn{3}{|l|}{ Arzt } \\
\hline Durchschnittliche Dauer der Aufnahme und Wundversorgung (min) & 18,4 & $11,41 €$ \\
\hline \multicolumn{3}{|l|}{ Pflege } \\
\hline $\begin{array}{l}\text { Durchschnittliche Dauer der Triage, Vorbereitung, Assistenz und Nach- } \\
\text { bereitung (min) }\end{array}$ & 28,5 & $11,97 €$ \\
\hline \multicolumn{3}{|l|}{ Summe Personal: $23,38 €$} \\
\hline Gesamtsumme: 44,12 & & \\
\hline
\end{tabular}

Notfallpauschale EBM 01212 zwischen 19:00 und 07:00 Uhr des Folgetages, zudem ganztägig an Samstagen, Sonntagen, gesetzlichen Feiertagen und am 24.12. und 31.12. [8, 9]. Die Abrechnung der eigentlichen Wundversorgung erfolgte unter der Pauschale EBM 02301 „Kleinchirurgischer Eingriff II und/oder primäre Wundversorgung mittels Naht“. Mit dieser Pauschale werden neben der Hautnaht unter Lokalanästhesie z.B. die Entfernung eines unter der Hautoberfläche gelegenen Fremdkörpers, die Deckung eines Hautdefektes sowie die Eröffnung eines subkutanen Abszess abgerechnet [10].

\section{Kostenkalkulation}

Die Fallkostenkalkulation erfolgte in Zusammenarbeit mit dem operativen Controlling. Es wurde eine Aufschlüsselung der anfallenden Einzelposten erstellt (• Tab. 1). Die Berechnung der Personalkosten erfolgte getrennt für Ärzte und Pflegepersonal nach ihren spezifischen Entgelten. Die Minutenkosten für das Personal wurden auf Basis der ab 20.02.2018 geltenden Entgelttabelle nach Tarifvertrag für Ärztinnen und Ärzte an den hessischen Universitätskliniken (TV-Ärzte Hessen 2018) berechnet. Bei einer 42-Stunden-Woche wurden für den Notaufnahmedienst (Durchschnittsgehalt aus 1./4./7. Weiterbildungsjahr) die Minutenentgelte und der Arbeitgeberanteil für die Lohnnebenkosten im Jahr 2018 mit 19,375\% berechnet. In den Lohnnebenkosten wurden die Renten-, Arbeitslosen-, Kranken- und Pflegeversicherung für einen imaginären Arbeitnehmer mit Lohnsteuerklasse 1, gesetzlicher Krankenversicherung und ohne Kinder erfasst. Die Nettojahresarbeitsstunden wurden unter der Berücksichtigung einer Ausfallzeit von 2,1\% für Ärzte berechnet [11]. So konnte ein Entgelt von $0,62 € / \mathrm{min}$ festgestellt werden. Der Minutenpreis des Pflegepersonals wurde anhand des Tarifvertrags für die Johann-Wolfgang-Goethe-Universität Frankfurt am Main (TV-G-U) in der Fassung des Änderungstarifvertrages vom 11.09.2017 (Entgelttabelle für Pflegekräfte, gültig $\mathrm{ab}$ 01.01.2018) für 38,5 Wochenstunden ermittelt. Als Referenz diente die vorwiegend beteiligte Entgeltgruppe P8. Die durchschnittlichen Personalkosten für die Stufen 3 und 4 wurden gemittelt. Der Arbeitgeberanteil der Lohnnebenkosten wurde entsprechend berechnet und eine Ausfallzeit von $6,85 \%$ berücksichtigt [11]. Auf diese Weise wurde ein Minutenentgeld von $0,42 € / \mathrm{min}$ errechnet.

Die Kosten für Verbrauchsmaterial, Desinfektionsmittel und Lokalanästhetikum und weiteres Nötige (• Tab. 1) wurden über unseren zentralen Einkauf bzw. die Klinikapotheke erhoben, hier sind die Einkaufspreise aufgeführt.

Die Wundversorgung wurde für alle Patienten entsprechend der S1-Leitlinie durchgeführt und die durchschnittliche Behandlungsdauer einer Standardwundversorgung mittels einschichtiger Hautnaht zugrunde gelegt, hierzu erfolgte eine Messung der durchschnittlich notwendigen Zeit für die Wundversorgung auf Seiten der Pflege und des ärztlichen Dienstes. Etwaige zusätzliche Leistungen der Radiologie oder des Labors wurden nicht berücksichtigt.

Die Kosten der einzelnen Faktoren wurden zu einem Gesamtkostenbetrag addiert und anhand der prozentualen Fallverteilung mit der Gesamtvergütung nach EBM verglichen (• Tab. 2).

\section{Ergebnisse}

Über das HIS konnten 724 Patienten, welche zwischen dem 01.01.2018 und dem 31.12.2018 mit einer Kopfplatzwunde in der Notaufnahme vorstellig wurden, identifiziert werden. In allen Fällen erfolgte eine Wundversorgung mittels Naht. Patienten mit kleineren Wunden, welche nicht chirurgisch wundversorgt werden mussten, wurden im Vorfeld aussortiert. Es zeigte sich eine vornehmlich männliche $(68,2 \%, 495 / 724)$ Kohorte jüngeren Alters $(42 \pm 21,5$ Jahre). 
Tab. 2 Kosten und Erlöse für die Wundversorgung einer Kopfplatzwunde mittels Naht

\section{Kosten}

Personalkosten

Materialkosten

Summe:

Erlöse

\begin{tabular}{|c|c|c|c|}
\hline EBM 02301 nach GOÄ Hessen & $13,96 €$ & 724 Pat & - \\
\hline EBM 01210 nach GOÄ Hessen & $12,99 €$ & 202 Pat & - \\
\hline EBM 01212 nach GOÄ Hessen & $21,10 €$ & 522 Pat & - \\
\hline \multicolumn{2}{|c|}{$($ EBM $02301 * 724+[$ EBM $01210 * 202+$ EBM $01212 * 522]) / 724$} & $\varnothing$ & - \\
\hline \multicolumn{3}{|c|}{ Summe: } & $32,80 €$ \\
\hline \multicolumn{3}{|l|}{ Gesamterlös pro Fall: } & $-11,32 €$ \\
\hline \multicolumn{3}{|l|}{ Kosten-Erlös-Defizit } & $-8195,68 €$ \\
\hline
\end{tabular}

\section{Vorstellungszeiten}

Es zeigte sich eine gehäufte Vorstellung von Patienten mit Kopfplatzwunden am Wochenende (39,2\%,284/724) Die meisten Vorstellungen erfolgten am Sonntag (20,7\%, 150/724). Knapp über die Hälfte $(50,1 \%, 369 / 724)$ wurde dabei während der Nachtzeit zwischen 19:00 und 7:00 Uhr behandelt, die meisten zwischen 20:00 Uhr und 21:00 (8,7\%, 63/724). Die Patientenanzahl an den jeweiligen Tagen bzw. zu den jeweiligen Uhrzeiten können den - Abb. 1 und 2 entnommen werden.

Im untersuchten Zeitraum stellten sich $202(27,9 \%)$ Patienten innerhalb der Regelarbeitszeiten von Montag bis Freitag zwischen 7:00 und 19:00 Uhr vor (EBM 01210) und wurden mit 120 Punkten und somit mit $12,99 €$ nach der Gebührenverordnung für Ärzte (GOÄ) in Hessen abgerechnet.

Insgesamt 522 Patienten (72,1\%) stellten sich dementsprechend nachts und/ oder am Wochenende vor (EBM 01212) und wurden mit 195 Punkten, entsprechend $21,10 €$ (GOÄ Hessen) bewertet. Für die Durchführung einer Wundversorgung wurden nach EBM 02310 13,96€ (129 Punkte, GOÄ Hessen) abgerechnet.

\section{Erlöse}

Der Gesamterlös für eine Wundversorgung tagsüber unter der Woche berechnet sich auf 26,95€ und pro nächtlichen Notfall bzw. am Wochenende oder an Feiertagen auf 35,06€. Bei 202 Fällen nach EBM 01210 entspricht das 5443,90€ für die Behandlung von Kopfplatzwunden unter der Woche. Die 522 nachts und am Wochenende (EBM 01212) durchgeführten Behandlungen erzielten Einnahmen von $18.301,32 €$. Somit errechnet sich ein Gesamterlös von 23.745,22€ für den retrospektiv untersuchten Zeitraum von Januar 2018 bis Dezember 2018 (12 Monate).

\section{Kosten}

Die Kostenkalkulation für Personal erfolgt wie im Absatz Material und Methoden beschrieben und in - Tab. 1 aufgeführt. Für die Personalkosten der Ärzte im Dienst wurden $0,624 € / \mathrm{min}$ berechnet. Für das Pflegepersonal wurde ein Minutenentgelt von $0,412 €$ berücksichtigt. Der durchschnittliche Zeitaufwand des ärztlichen Personals für eine Wundversorgung inklusive der eingehenden Anamnese, Untersuchung, Beratung bzw. Aufklärung, Behandlung sowie die abschließende Erstellung eines Arztbriefs und Ausstellen von Rezepten und ggf. Krankmeldung belaufen sich auf durchschnittlich 18,4 min. Die assistierende Pflege hat einen Zeitaufwand von 28,5 min durch die vorherige Triage, $\mathrm{Pa}$ tienten- und Materialvorbereitung sowie die Verbandanlage und Nachbereitung des Behandlungsraumes. Damit entstehen $23,38 €$ Gesamtpersonalkosten, anteilig $11,41 €$ für die ärztliche und $11,97 €$ für die pflegerische Leistung.

Die Verbrauchsmaterialien kosten pro Wundversorgung 20,74€ (• Tab. 1). Kosten für Reinigung, Verwaltung und Geräte- und andere Vorhaltekosten wurden nicht berechnet.

\section{Kosten-Erlös-Verhältnis}

Es entstehen pro versorgten Patienten Gesamtkosten von 44,12€. Dem gegenübergestellt wurde die durchschnittliche Vergütung von $32,80 €$ pro Fall.

Nach Abzug der Kosten von den Erlösen gegenüber den Krankkassen ergibt sich ein Defizit von $11,32 €$ pro Fall. Bei einem Aufkommen von 742 Patienten mit einer Kopfplatzwunde im Jahr 2018 entspricht dies einem Jahresdefizit von $8195,68 €$ (• Tab. 2).

\section{Traumamechanismen}

Die häufigste Traumaursache für eine Kopfplatzwunde war in unserer Kohorte das Bagatelltrauma (30,2\%, 219/724), also ein Anpralltrauma des Kopfes an stumpfen Gegenständen wie z. B. an Fensterkanten, Möbeln oder an aus geringer Höhe auf den Kopf herabfallendekleinere Gegenstände.

Ein nicht zu vernachlässigender Anteil unserer Patienten stellten sich nach einer körperlichen Auseinandersetzung oder Körperverletzung vor $(20,9 \%, 151 / 724)$. Der dritthäufigste ursächliche Traumamechanismus in unserer Kohorte war der Stolpersturz mit konsekutivem Kopfanprall mit 17,0\% (123/724) aller Vorstellungen (• Abb. 3).

\section{Alkohol und Kopfplatzwunden}

Unter den 724 Patienten, welche 2018 mit einer Kopfplatzwunde in unserer Zentralen Notaufnahme vorstellig wurden, erfolgte bei 268 (37,0\%) die Dokumentation einer Alkoholintoxikation.

\section{Diskussion}

Bei einer nach $\$ 108$ SGB V gegebenen Verpflichtung der zugelassenen Krankenhäuser Notfallversorgung zu betreiben, ergibt sich anhand des Beispiels einer Gegenüberstellung von Erlösen und Kosten der ambulanten chirurgischen Versorgung von Kopfplatzwunden in der Vergütung nach EBM ein deutliches Defizit. Bereits seit Jahren ist das bekannte Defizit zwischen den deutlich zu hohen Kosten der Notfallversorgung in Deutschlands Krankenhäusern 


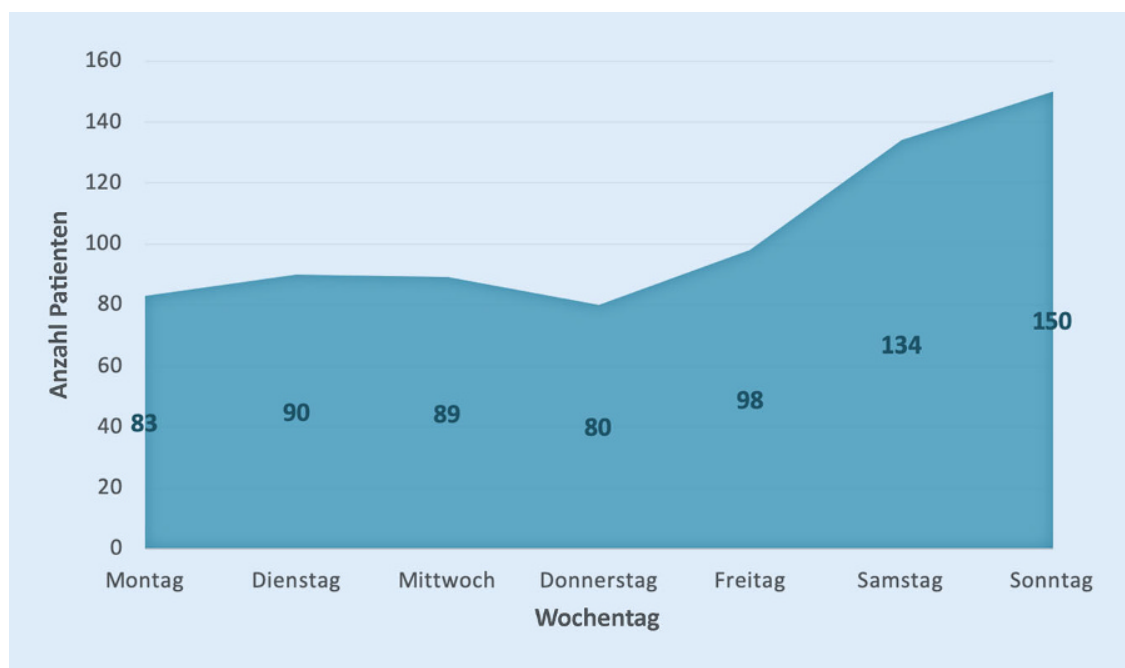

Abb. $1 \Delta$ Anzahl der Patienten im Verlauf der Woche

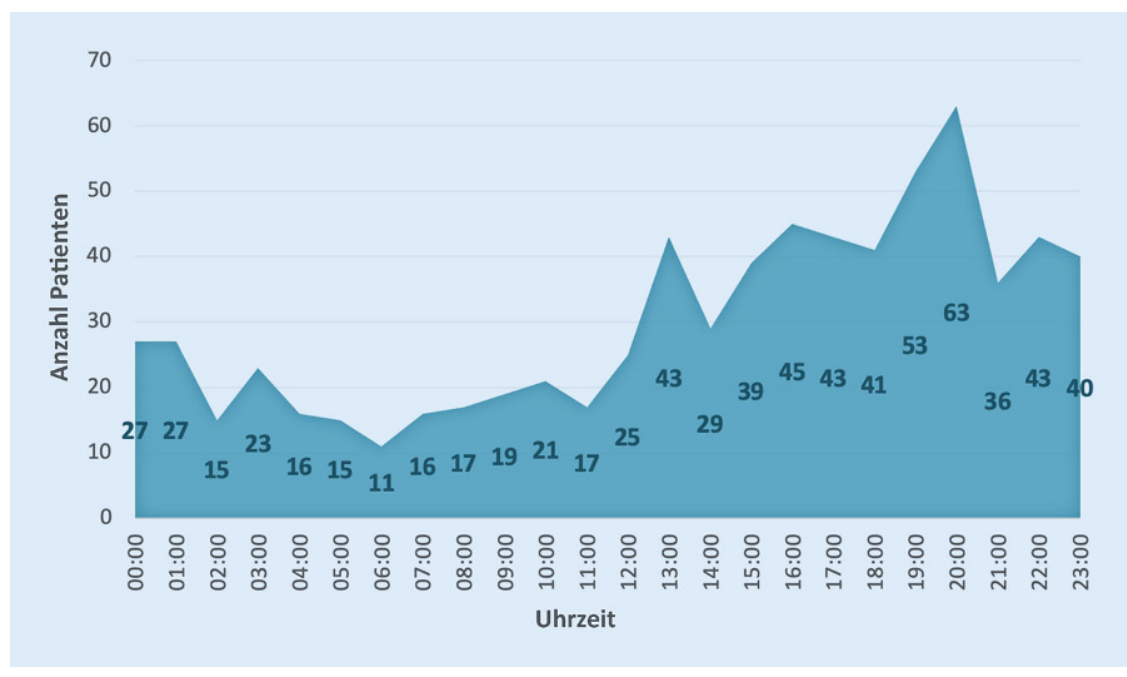

Abb. $2 \Delta$ Anzahl der Patienten im Tagesverlauf

Thema wichtiger Diskussionen. Dabei muss einerseits die geringe Vergütung der Notfallversorgung, aber auch das zunehmend hohe Patientenaufkommen in einer für jedermann jederzeit frei zugänglichen Notaufnahme thematisiert werden. In einem System, welches von zunehmender Knappheit der verfügbaren Ressourcen geprägt ist, wird auch die Organisation der stetig zunehmenden Nachfrage nach medizinischer Notfallbehandlung sowie dessen Finanzierbarkeit zunehmend schwierig. Um keine Abstriche in der Verfügbarkeit und Qualität der Versorgung zu befürchten, muss bei Zunahme der Behandlungsfälle die Wirtschaftlichkeit der einzelnen
Leistungen analysiert und optimiert werden.

\section{Regredienz von Notfällen im kas- senärztlichen Bereitschaftsdienst}

Der starken Regredienz an Notfällen im kassenärztlichen Bereitschaftsdienst von 2009 bis 2015 um 15\% steht die stetig steigende Zahl der ambulanten Notfälle im Krankenhaus gegenüber. Dabei zeigte sich ein Anstieg der Fälle von 6 Mio. im Jahr 2009 auf über 8,5 Mio. im Jahr 2015 [17]. Diese Steigerung um 42\% verdeutlicht die Verschiebung der Notfallvorstellung in die Notaufnahme [1, 13, 17]. Die Frage der Finanzierbarkeit der Notaufnahmen wird damit zuneh- mend wichtig. Um dieser gerecht zu werden und um Vergütungsdefizite offen $\mathrm{zu}$ legen, werden einzelne Leistungen anhand ihrer Wirtschaftlichkeit analysiert [7]. Bisher durchgeführte Untersuchungen konzentrierten sich vor allem auf besondere Krankheitsbilder, polytraumatisierte Patienten oder bildeten die Gesamtkosten der ambulanten Notfallversorgung ab [12, 21, 22, 25-27]. Für die allgemeine Notfallversorgung konnte ein fallbezogener Erlös von $32 €$ bei durchschnittlichen Kosten von $120 €$ aufgezeigt werden. Daraus resultierte ein Fehlbetrag von $88 €$ pro Fall $[14-16,24]$. Anhand dieser Arbeit konnte am Beispiel der Versorgung einer einfachen Kopfplatzwunde, die in jeder Klinik häufig durchgeführt wird, ein Vergütungsdefizit von 11,32€ gezeigt werden.

Rund die Hälfte (52\%) der 2 Mio. Verletzten, welche sich in den Notaufnahmen deröffentlichen Krankenhäuservorstellt, verbleibt ambulant. Dabei erfolgt die Abrechnung von $80 \%$ der Fälle über das EBM-System mittels GKV [18].

Nach dem Projektbericht der Kassenärztlichen Bundesvereinigung zur Notfallversorgung in Deutschland vom April 2018 steigt die Anzahl der ambulanten Notfälle am Wochenende um etwa $39 \%$. Die meisten ambulanten Notfallbehandlungen werden werktags in der Zeit zwischen 18 und $20 \mathrm{Uhr}$ durchgeführt und am Wochenende zwischen 10 und 12 Uhr [17]. Wie erwartet belegt auch diese Studie die vermehrte Vorstellung von Notfallpatienten mit Kopfplatzwunden außerhalb der regulären Öffnungszeiten der niedergelassenen Ärzte, nämlich hauptsächliche am Wochenende, besonders sonntags, sowie zwischen 18 und 22 Uhr. Insgesamt stellten sich lediglich 27,9\% der Patienten Montag bis Freitag zwischen 7 und 19 Uhr und damit 72,1\% entweder nachts oder am Wochenende vor.

\section{Probleme durch die Abrechnung nach EBM}

Inhalt und Umfang anrechnungsfähiger Leistungen im Bereich der vertragsärztlichen Versorgung werden auf Kalkulationsgrundlage der betriebswirtschaftlichen Aufwandserfassung im niederge- 


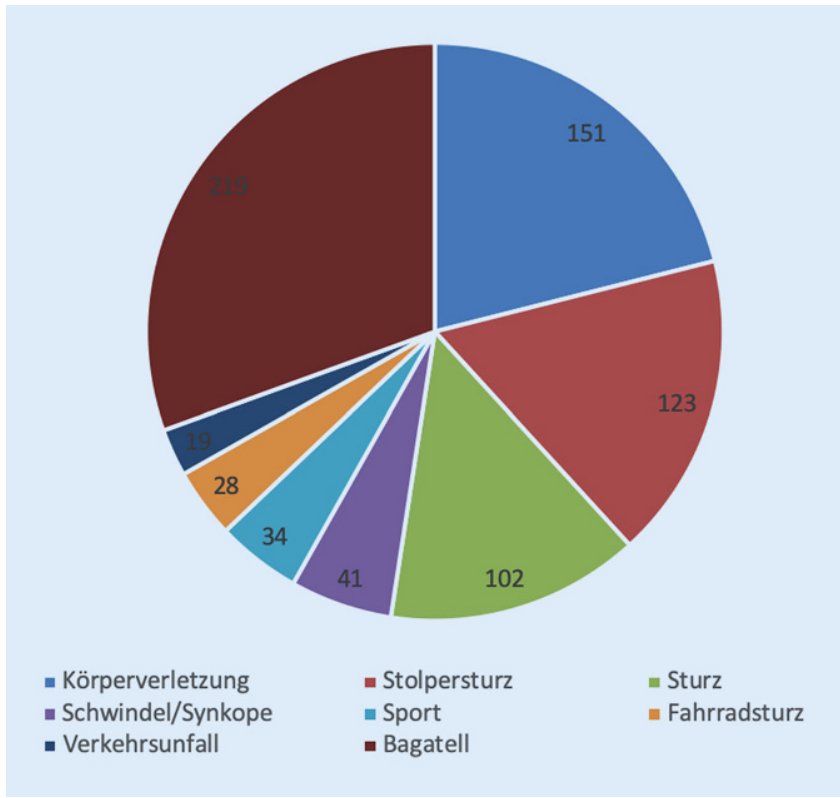

\section{Großer Anteil an Patienten mit Alkoholeinfluss}

Einen nicht zu vernachlässigenden Anteil der in der Notaufnahme mit einer Kopfplatzwunde vorstelligen Fälle stellen unter Alkoholeinfluss stehende Patienten dar; in unserer Kohorte lag dieser Anteil bei 37,0\%. Diese Patienten sind bekanntermaßen schwer einschätzbar, da die Menge und der Zeitpunkt der Einnahme meist unbekannt bleiben. Bei steigenden Fallzahlen an alkoholisierten Patienten in deutschen Notaufnahmen werden Ärzte und das Pflegepersonal vor allem nachts und am Wochenende zunehmend gefordert und auch zeitlich gebunden [23]. Die Alkoholintoxikation ist dabei ein eigenständiges Krankheitsbild, welches ein gut ausgebildetes Team fordert und einen hohen Mehraufwand bedeutet. Nicht nur die Anamnese und Untersuchung, sondern auch die Versorgung ist in dieser Patientengruppe deutlich erschwert. Diese erschwerenden Umstände, die nicht nur eine vermehrte Ressourcenbindung, sondern auch einen deutlich vergrößerten Zeitaufwand bedeuten, werden in der aktuellen Vergütung der Notfallversorgung nicht abgebildet und stellen einen zusätzlichen relevanten Faktor für Kostendefizite für die Notaufnahme dar [19, 20].

\section{Limitationen}

Im komplexen Umfeld einer Klinik konnten nicht alle Faktoren erfasst werden. Wie bereits erwähnt, wurden Kosten für Verwaltungs-/Aufnahmepersonal, Reinigung, Abrechnung sowie die Zuschläge für Nacharbeit und anfallenden Freizeitausgleich nicht erfasst. Vorhaltekosten für Personal und Investitionskosten wurden ebenfalls nicht berücksichtigt. Jedoch kommt es in dieser Erhebung auch ohne die zuvor genannten Faktoren $\mathrm{zu}$ einer Minderdeckung bei der einfachen Versorgung einer Kopfplatzwunde. Es zeigte sich zudem bei der Auswertung der Daten nur ein niedriger Anteil an privat (14) und berufsgenossenschaftlich (23) abgerechneten Patienten in unserem Kollektiv von 2018. Die beispielhafte Versorgung eines privaten Patienten am Samstagnachmittag, welche mit einem 
2,3-fach berechneten Satz nach GOÄ einen Erlös von $35,78 €$ erzielt, ist daher in Bezug auf den Gesamtfehlbetrag vernachlässigbar. Gerade bei den privat versicherten Patienten scheinen andere Strukturen als die Notaufnahme, z. B. der privat ärztliche Notdienst, primäre Anlaufstrukturen zu sein.

\section{Fazit für die Praxis}

Die bisherige Abrechnung der ambulanten Wundversorgung in den Notaufnahmen der Krankhäuser durch den einheitlichen Bewertungsmaßstab ist höchst unzureichend. Eine Alternative oder Erweiterung des Vergütungssystems für den Krankhaussektor bzw. die Einführung einer konsequenteren Patientensteuerung scheinen unumgänglich, um der defizitären Finanzstruktur der Notaufnahmen entgegenzuwirken.

\section{Korrespondenzadresse}

Dr. med. Nils Mühlenfeld

Goethe Universität Frankfurt

Theodor-Stern-Kai 7, 60590 Frankfurt am Main, Deutschland

nils.muehlenfeld@kgu.de

Author Contribution. N. Mühlenfeld, C.R. Schindler und R.D. Verboket entwickelten die Idee für dieses Projekt und führten die statistische Analyse sowie die Datenerhebung durch. N. Mühlenfeld, C.R. Schindler, J. Sterz, P. Thönissen, P. Störmann, I. Marzi und R.D. Verboket verfassten den Artikel. Jeder Autor trug wichtige inhaltliche Aspekte bei und stimmte der Publikation des Artikels in der eingereichten Form zu.

Funding. Open Access funding provided by Projekt DEAL.

\section{Einhaltung ethischer Richtlinien}

Interessenkonflikt. N. Mühlenfeld, C.R. Schindler, J. Sterz, P. Thönissen, P. Störmann, I. Marzi und R.D. Verboket geben an, dass kein Interessenkonflikt besteht.

Für diesen Beitrag wurden von den Autoren keine Studien an Menschen oder Tieren durchgeführt. Für die aufgeführten Studien gelten die jeweils dort angegebenen ethischen Richtlinien.

Open Access. Dieser Artikel wird unter der Creative Commons Namensnennung 4.0 International Lizenz veröffentlicht, welche die Nutzung, Vervielfältigung, Bearbeitung, Verbreitung und Wiedergabe in jeglichem Medium und Format erlaubt, sofern Sie den/die ursprünglichen Autor(en) und die Quelle ordnungsgemäß nennen, einen Link zur Creative Commons Lizenz beifügen und angeben, ob Änderungen vorgenommen wurden.

Die in diesem Artikel enthaltenen Bilder und sonstiges Drittmaterial unterliegen ebenfalls der genannten Creative Commons Lizenz, sofern sich aus der Abbildungslegende nichts anderes ergibt. Sofern das betreffende Material nicht unter der genannten Creative Commons Lizenz steht und die betreffende Handlung nicht nach gesetzlichen Vorschriften erlaubt ist, ist für die oben aufgeführten Weiterverwendungen des Materials die Einwilligung des jeweiligen Rechteinhabers einzuholen.

Weitere Details zur Lizenz entnehmen Sie bitte der Lizenzinformation auf http://creativecommons.org/ licenses/by/4.0/deed.de.

\section{Literatur}

1. AQUA-Institut (2016) Ambulante Notfallversorgung - Analyse und Handlungsempfehlungen

2. Amelung VE (2010) Managed Care. Neue Wege im Gesundheitsmanagement

3. Ashley DW, Mullins RF, Dente CJ, Garlow L, Medeiros RS, Atkins EV, Ferdinand CH (2017) What are the costs of trauma center readiness? Defining and standardizing readiness costs for trauma centers statewide. Am Surg 83(9):979-990. Retrieved from https://www.ncbi.nlm.nih.gov/ pubmed/28958278

4. Benchimol El, Smeeth L, Guttmann A et al (2015) The reporting of studies conducted using observational routinely-collected health data (RECORD) statement. PLoS Med 12:e1001885

5. Bundesministerium für Gesundheit (2019) Einheitlicher Bewertungsmassstab EBM. https://www.bundesgesundheitsministerium. de/service/begriffe-von-a-z/e/einheitlicherbewertungsmassstab-ebm.html. Zugegriffen: 13.01.2020

6. von Elm E, Altmann DG, Egger M et al (2008) Das Strengthening the Reporting of Observational Studies in Epidemiology (STROBE-) Statement. Internist 49:688-693

7. Haas C, Larbig MST (2015) Gutachten zur ambulanten Notfallversorgung im KrankenhausFallkostenkalkulation und Strukturanalyse

8. Kassenärztliche Bundesvereinigung (2019) EBM Notfallpauschale 01210. https://www.kbv.de/ tools/ebm/html/01210_2902913022083955875 136.html.Zugegriffen: 13.01 .2020

9. Kassenärztliche Bundesvereinigung (2019) EBM Notfallpauschale 01212. https://www.kbv.de/ tools/ebm/html/01212_2904079587704631440 352.html.Zugegriffen: 13.01.2020

10. Kassenärztliche Bundesvereinigung (2019) EBM 02301 kleinchir. Eingriff II/Naht. https://www.kbv.de/tools/ebm/html/02301 2900461788052279415424.html. Zugegriffen: 13.01.2020

11. Hessisches Ministerium für Soziales und Integration (2017) Gesundheitsbericht Hessen. Hessisches Ministerium für Soziales und Integration, Wiesbaden

12. Muhlenfeld N, Sohling $N$, Marzi I, Pieper $M$, Paule E, Reif PS, Strzelczyk A, Verboket RD, Willems LM (2019) Fractures in Parkinson's Disease: Injury Patterns, Hospitalization, and Therapeutic Aspects. Eur J Trauma Emerg Surg. https://doi.org/ 10.1007/s00068-019-01240-z

13. Muhlenfeld N, Thoenissen P, Verboket R, Sader R Marzi I, GhanaatiS (2020) Combined Trauma in Cra- niomaxillofacial and Orthopedic-Traumatological Patients: The Need for Proper Interdisciplinary Care in Trauma Units. Eur J Trauma Emerg Surg. https:// doi.org/10.1007/s00068-020-01479-x

14. Qvick B, Bühren V, Woltmann A (2011) Ist ein Polytrauma heutzutage noch bezahlbar? Unfallchirurg 115(10):892-896

15. Schindler CR, Lustenberger T, Marzi I, Verboket RD (2020) Kostenfaktor „ambulante Wundversorgung" in der Notaufnahme: Kosten-Erlös-Defizit der ambulanten Wundversorgung in einer deutschen Universitatsklinik. https://doi.org/10.1007/ s00113-020-00819-1

16. Schmelz A, Ziegler D, Beck A et al (2002) Akutstationäre Behandlungskosten polytraumatisierter Patienten. Unfallchirurg 105:1043-1048

17. Schmidt CM, Bauer TK, Rumpf S (2018) Notfallversorgung in Deutschland Projektbericht im Auftrag der Kassenärztlichen Bundesvereinigung

18. Statistisches Bundesamt (2016) Diagnosedaten derPatienten und Patientinnen in Krankenhäusern (einschl. Sterbe- und Stundenfälle) - Fachserie 12 Reihe 6.2.1

19. Somasundaram R, Wrede C (2016) Notfallversorgung von Patienten mit alkoholbedingten Erkrankungen. Notfall Rettungsmed 19:3-3

20. springermedizin.de (2016) Akute Alkoholintoxikation. https://www.springermedizin. de/notfallmedizin/wissen-macht-arzt/akutealkoholintoxikation/8710106.Zugegriffen:6. Febr. 2020

21. Verboket RD, Muhlenfeld N, Sterz J, Stormann $P$, Marzi I, Balcik Y, Rosenow F, Strzelczyk A, Willems LM (2020) Stationare Versorgungskosten, kostenverursachende Faktoren und potenzielle Vergutungsprobleme bei Verletzungen und Frakturen im Rahmen epileptischer Anfalle. Chirurg. https://doi.org/10.1007/s00104-02001257-w

22. Verboket RD, Söhling N, Marzi I et al (2019) Prevalence, risk factors and therapeutic aspects of injuries and accidents in women with epilepsy. Eur JTrauma Emerg Surg 45:375-381

23. Verboket R, Söhling N, Schmitz L et al (2019) Violence in the emergency department of a maximum care hospital. Chirurg 90:570-575

24. Verboket R, Verboket C, Schöffski O et al (2019) Costs and proceeds from patients admitted via the emergency room with mild craniocerebral trauma. Unfallchirurg 122:618-625

25. Verboket RD, Muhlenfeld N, Woschek M, Marzi I, Pieper M, Zollner JP et al (2020) Inpatient treatment costs, cost-driving factors and potential reimbursement problems due to fall-related fractures in patients with Parkinson's disease. Chirurg 91(5):421-427

26. Willems LM, Richter S, Watermann N et al (2018) Trends in resource utilization and prescription of anticonvulsants for patients with active epilepsy in Germanyfrom 2003 to2013-A ten-yearoverview. Epilepsy Behav 83:28-35

27. Willems LM, Watermann N, Richter S, Kay L, Hermsen AM, Knake S et al (2018) Incidence, Risk Factors and Consequences of Epilepsy-Related Injuries and Accidents: A Retrospective, Single Center Study. Front Neurol 9:414 
Hier steht eine Anzeige.

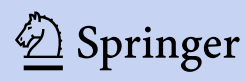

